\title{
Research on Crowdsourcing Camera Mode System based on Block Chain Technology
}

\author{
Dou $\mathrm{Hu}^{1}$, Chuang $\mathrm{Ma}^{1}$, Yuanlong Gao ${ }^{1}$ and Wenlong Liu ${ }^{1}$ \\ ${ }^{1}$ School of Information and Communication Engineering, Dalian University of Technology, Dalian, China
}

\begin{abstract}
As a new business model, the crowdsourcing camera mode has been paid close attention in recent years. However, traditional crowdsourcing operations rely too much on centralized institutions, which have many drawbacks. It is precisely because of this that the superiority of block chain technology is reflected. With the flourishing development of the Internet and the ever-changing block chain technology, this paper provides the research on crowdsourcing camera mode based on block chain technology from four aspects including the analysis of decentralization, the strengthen of security precautions, the establishment of appropriate systems and the gradually transition of token system.
\end{abstract}

\section{INTRODUCTION}

In recent years, the rise of the Internet has led to the development of a large number of emerging industries, among which the 'photographing and making money' is a self-service crowdsourcing service model under the mobile Internet that is also known as the crowdsourcing camera mode. The users first download the applications (APPs) of the crowdsourcing camera mode, then register as a member of the APP, and then receive the tasks of taking a picture, and earn a reward for accomplishing the tasks. The crowdsourcing service model provides enterprises with various business inspections and information collection. Compared with traditional market survey methods, it can greatly reduce survey costs, and effectively guarantee the authenticity of survey data and shorten the survey period.

However, the trust between people, operating costs, and redundant central institutions are all obstacles to this approach. If the crowdsourcing service model is operated in a traditional commercial manner, there is bound to be a central agency [1]. Hence, the photographing tasks will be transferred through the central agency. Moreover, the establishment of a large-scale trading platform not only increases the operating cost of crowdsourcing services and the response time to events, but also presents a great security risk. Considering the characteristics of the block chain technology, including decentralization, high security, strong scalability, and low transaction costs, this paper researches a new operating plan based on block chain technology for crowdsourcing camera mode.

The rest of the paper is organized as follows. In section II, we introduce the traditional crowdsourcing camera mode. The research of crowdsourcing service system model based on the block chain technology is given in section III. Finally, section IV ends the paper with the conclusion.

\section{THE TRADITIONAL CROWDSOURCING CAMERA SERVICE}

The architecture of traditional the crowdsourcing camera mode can be summarized as shown in Fig 1 .

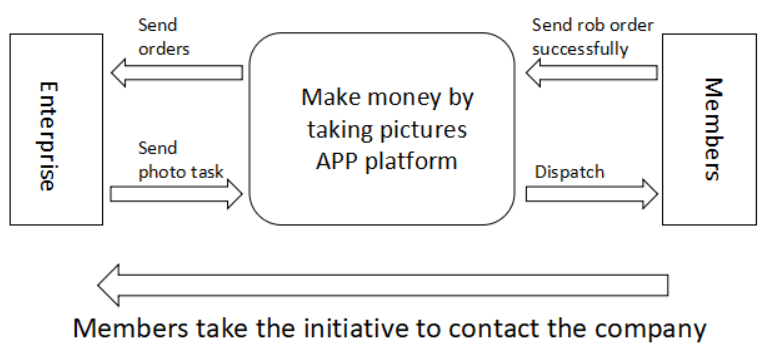

Fig 1: The architecture of traditional the crowdsourcing camera mode

From Fig 1, the app serves as a third-party trading platform, which is the hub of the transaction. However, there are many security risks in traditional crowdsourcing. The first is the issue of trust. For this mode, tasks are distributed through the APP, so the

*Corresponding author: Wenlong Liu: liuwl@dlut.edu.cn 
number of people receiving the tasks is large, and the large number of people will lead to uneven quality of professionalism, such as identity fraud, perfunctory tasks, once the platform is unable to identify true or false, it will create a crisis of confidence. What is more, due to the different amount of tasks and the degree of difficulty in photographing, it is easy for people to lose trust in the platform. Second is the security issue. Because the platform collects a large amount of information about the transaction and even includes private information, once the information is leaked, the platform system will be compromised. The third problem is miscellaneous issues. The redundancy of platform information is mixed, and the recipient cannot distinguish authenticity from others. This can lead to inefficiencies in the operation process. In other words, the participation of third-party platforms will lead to low levels of effectiveness.

In order to address the above issues, the crowdsourcing service system model based on the block chain technology are proposed which is introduced in next section.

\section{CROWDSOURCING CAMERA SYSTEM BASED ON BLOCK CHAIN TECHNOLOGY}

\subsection{More reasonable decentralization}

Considering the issues of the trust, security, and miscellaneous in the traditional centralized operation model, which is combined with information security, which inevitably represents its existence risk. For this regard, the block chain technology can be an available solution, due to the characteristic of decentralization. The "centralization" in the block chain is a distributed data storage structure for the purpose of protecting the security of information. Firstly, due to the open and transparent distributed storage characteristics of block chains, it can adopt distributed storage based on Peer to Peer (P2P) networks where each node saves one data backup. Hence, the trust degree is greatly improved. Then the block chain has features that cannot be modified and can be traced, and security is fully guaranteed. Finally, block chain technology is decentralized, decentralizing information to both sides of the transaction, making information communications more efficient, reducing operating costs, providing faster response time, and greatly improving efficiency.

In the case of cross-border payments and exchange systems as an example [2], we illustrate the cost saving effect of centralization and the improvement of system security. Traditional cross-border payment and exchange systems rely on centralized intermediary credit to connect different entities in the financial market. This scheme requires the reduction of information asymmetry and the trust mechanisms established by the authority to achieve the value transfer and all participants unanimously recognized the central authority with absolute credibility. This will lead to high costs, increase the time of payment exchange process, weaken the liquidity of funds, and there are also payment and system risks in terms of security.

However, the situation will change when the centralization is abandoned and adopt the logic of decentralization in block chain technology. On the one hand, decentralization avoids the breakdown of the entire system caused by the attack that central organization suffered. Benefit by the existence of distributed ledgers, each node has a complete copy of the ledger, and the failure of some nodes is insufficient to affect the entire network. On the other hand, it reduces the cost of establishing a third-party central hub. It has become an efficient and low-cost global block chain network financial transmission protocol. According to McKinsey's calculations, this solution will reduce the cost of each transaction from US\$26 to US $\$ 15$ and save about $42.3 \%$ of the cost. According to China's 2015 cross-border payment and settlement of approximately 800 billion Yuan, we will save 336 billion Yuan every year.

\subsection{Strengthen safety precautions}

The system of block chain cannot be deleted and cannot be changed. Each node in the chain is central. The block chain has its unique features in terms of security.

Through P2P download, P-CDN distributed storage and distributed computing. A large amount of data has been distributed to countless computers to achieve packaging, disperse the danger in centre and increase efficiency.

JAVA can be used to achieve a simple block chain design [3]. Specifically, the 'crypto-js' library can be used to create a block and the block chain class can connect the block, and define the last block of the hash. In this way, a simple block chain is generated. Of course, it can be optimized using Proof of Work (POW) or P2P networks. POW can be used to prevent misuse and defence against attacks through a simple amount of calculations. The incentive measure is to generate a mining mechanism that can be generated using the function known as 'minePendingTransactions' in JAVA. 
In this case, information is difficult to modify. It is highly distributed and it reduces external risks. So block chain technology is very good at controlling information security.

In summary, two proposals are proposed for the crowdsourcing service mode secure encryption method as follow:

1.Using the Tech Target and session key consultation mechanisms based on block chain [4]. The identity-based encryption algorithm IBE can use the user's identity as a public key for encryption or signature verification. To avoid the loss of public keys and result in security issues and to prevent replay attacks, the public key needs to be updated regularly.

2.Similar to food traceability technology, we provide customers with tracking services system. The tracking services system includes physical, communications, database and interface layers. Among them, block chain technology is applied to the database layer and communication layer. The physical layer mainly includes smart sensors and actuators for data acquisition and forwarding to the upper layer protocol, which is substantially similar to the physical layer in the structure of the Internet of Things. The communication layer of the traceability system uses Ethernet platform based on block chain technology and hash information transfer protocol to prevent the attacked node from destroying the system through the network. In the e-commerce environment, the frequency of transactions, the area involved, and the number of commodities have increased substantially [5]-[6]. Incomplete and asymmetric information among various entities has increased the risk of monetary payment links.

In 2016, the third-party Internet payment transaction in China reached 19.2 trillion Yuan, a year-on-year increase of $62 \%$. In the face of the increasing number of Internet transactions, the security construction of the payment link has become the key. In the block chain technology, transaction data cannot be tampered with the characteristics of transaction traceability. It can be used as a simple and effective tracking tool to jointly maintain a reliable database. To the utmost extent it guarantees the security of the transaction process.

\subsection{Establish the appropriate systems}

In addition to Bitcoin and Ethernet, a number of systems related to block chain have emerged in recent years which can be used for the crowdsourcing model. Hyperledger is an open source project launched by the Linux Foundation in 2015 that promotes block chain digital technology and transaction verification [7]-[8].
Because of the characteristics of the peer-to-peer network in hyperledger, distributed ledger technology is completely shared, transparent, and decentralized, and is well-suited for node-network-based business model applications. hyperledger has identity and auditability, private transactions and confidentiality contracts, users can choose their own consensus algorithms and write chain code (also known as: smart contracts) performance, and scalability through mainstream programming languages.

In the research and development of block chain application projects for the photo-financing and crowd-sourcing project, the research and improvement of consensus algorithms is very important. Therefore, the development environment based on the hyperledger can be used not only for programming development of enterprise-level application projects, but also for performance analysis tests of related consensus algorithms, smart contracts, and the like. Taking the Hyperledger development environment as an example, we have integrated the following system building steps:

Step 1: Build a test environment on a Linux system. According to the network environment construction plan of the four verification nodes given on the official website, the Docker installation test environment is used in the virtual machine.

Step 2: Build a test network. Multiple dockers are installed on one PC to carry multiple authentication nodes; multiple dockers are installed on multiple PCs to achieve communication between dockers so as to expand the network scale.

Step 3: View the consensus module, analyze the source code. Understand the writing specification format of the consensus code and find the relevant event interface.

Step 4: Determine the boot interface and consensus event flow of the consensus module. Find the boot consensus algorithm interface function to find all the parameters and related parameters for the consensus algorithm in the system.

Step 5: Change the original PBFT consensus code, replace and test the validity of the consensus algorithm.

In summary, by properly constructing a suitable system such as hyperledger, a crowdsourcing camera model can be better operated.

\subsection{Gradual transition to the token system}

The crowdsourcing service model of the 'photographs and making money' is still an emerging niche industry. Therefore, in the current stage, efforts should be made to strengthen the publicity of such crowd-sourcing 
models and enhance the crowd-sourcing model's influence and popularity.

After the crowdsourcing operation model is more popular, it should be gradually transformed from the original common currency system to the token system. Using tokens such as Bitcoin [9] as a reward for rewards, there is no application at the beginning of the application of the token system, but only the currency equal to the exchange of labour for the replacement of the currency. The currency then shows its value elsewhere and is used as a currency of circulation. In the second phase, new applications emerged on this platform and new smart contracts emerged. At this time, there is a new possibility. Tokens can gain access to applications that come from their own platform, and then they will turn around in this sector. Then they must reward those who provide value for their uses. This forms a closed loop.

\section{CONCLUSIONS}

The generation of crowdsourcing camera models such as photo-taking and making money will further promote the development of the Internet and block chain. Through analysis, it can be seen that block chain technology has some obvious advantages for crowdsourcing operations, but at the same time, there are also many challenges. For the problems that may be foreseen, we have given the above suggestions for improvement.

\section{REFERENCES}

1. Axelrod, R., Cohen, M. Harnessing Complexity [J]. The Free Press, New York, 1999.

2. Halder, P., Das, S. H. JaSTA-2: Second version of the Java Superposition T-matrix Application [J]. Computer Physics Communications, 2017.

3. Agrawal, A., Catalini, C. and Goldfarb, A. The Geography of Crowd funding [J]. Social Science Electronic Publishing, 2011.

4. Armstrong, M., Wright, J. Two-Sided Markets, The New Palgrave Dictionary of Economics [M]. Basingstoke: Palgrave Macmillan, 2008

5. Nakamoto, S. Bitcoin: A peer to peer Electronic Cash System [EB/OL], 2016.

6. Nakamoto, S. Bitcoin: A peer-to-peer electronic cash system [OL]. https://bitcoin.org/bitcoin.pdf, 2008.

7. Aiello, D, Luca, D.D., Gionfriddo, E., Naccarato, A., Napoli, A., Romano, E., Russo, A., Sindona, G. and Tagarelli, A. Review: Multistage mass spectrometry in quality, safety and origin of foods [J]. European Journal of Mass Spectrometry, 2011, 17(1):2-3.
8. Satoshi N. Bitcoin: A peer-to-peer electronic cash system, 2008. https://bitco.in/pdf/bitcoin.pdf

9. Hyperledger [EB/OL], 2017. https: //www.hyperledger. org/ 